\title{
Article \\ Effect of Excitation Wavelength on Optical Performances of Quantum-Dot-Converted Light-Emitting Diode
}

\author{
Caiman Yan ${ }^{1}$, Xuewei $\mathrm{Du}^{1}$, Jiasheng $\mathrm{Li}^{1}$, Xinrui Ding ${ }^{1}$, Zongtao $\mathrm{Li}^{1}{ }^{1,2}, \mathbb{C}$ and Yong Tang ${ }^{1}$ \\ 1 Key Laboratory of Surface Functional Structure Manufacturing of Guangdong High Education Institutes, \\ South China University of Technology, Guangzhou 510641, China \\ 2 Foshan Nationstar Optoelectronics Company, Ltd., Foshan 528000, China \\ * Correspondence: meztli@scut.edu.cn; Tel.: +86-138-2446-0886
}

Received: 10 July 2019; Accepted: 25 July 2019; Published: 1 August 2019

check for updates

\begin{abstract}
Light-emitting diode (LED) combined with quantum dots (QDs) is an important candidate for next-generation high-quality semiconductor devices. However, the effect of the excitation wavelength on their optical performance has not been fully explored. In this study, green and red QDs are applied to LEDs of different excitation wavelengths from 365 to $455 \mathrm{~nm}$. The blue light is recommended for exciting QDs from the perspective of energy utilization. However, QD LEDs excited at $365 \mathrm{~nm}$ have unique advantages in eliminating the original peaks from the LED chip. Moreover, the green or red light excited by ultraviolet light has an advantage in colorimetry. Even for the $455 \mathrm{~nm}$ LED with the highest QD concentration at $7.0 \mathrm{wt} \%$, the color quality could not compete with the $365 \mathrm{~nm}$ LED with the lowest QD concentration at $0.2 \mathrm{wt} \%$. A 117.5\% (NTSC1953) color gamut could be obtained by the $365 \mathrm{~nm}$-excited RGB system, which is $32.6 \%$ higher than by the $455 \mathrm{~nm}$-excited solution, and this can help expand the color gamut of LED devices. Consequently, this study provides an understanding of the properties of QD-converted LEDs under different wavelength excitations, and offers a general guide to selecting a pumping source for QDs.
\end{abstract}

Keywords: colorimetry; excitation wavelength; light-emitting diode; quantum dots

\section{Introduction}

Light-emitting diodes (LEDs) are fundamental devices for backlights and displays [1,2]. Nowadays, the common base pixel is composed of red, green, and blue (RGB) tricolor in both RGB backlights and direct display technology. Three different-color epitaxial chips are mainly applied in an RGB pixel: Blue, green, and red LED chips [3]. However, this solution cannot avoid the following problems: First, the blue, green, and red LEDs have different turn-on voltage characteristics, resulting in voltage balance problems in the circuit design [4]; second, owing to process limitations, the optical efficiency and production yield of red LEDs cannot compare with blue LEDs [5], resulting in high technology thresholds and increased costs; third, it is difficult to further improve the color quality in the current common RGB pixel because of the epitaxial material property limitation.

In order to solve the above difficulties, there are two advanced technologies under discussion. The first advanced technology is using only one LED chip, such as a blue or ultraviolet chip, to solve the voltage mismatch problem [6,7]. For the blue chip solution, the blue light is from a blue LED chip; the green and red light are obtained from the blue light to excite the green and red fluorescent materials. For the ultraviolet chip solution, the blue, green, and red light are all obtained from the ultraviolet-light-excitation fluorescent material [8]. Another technique is to maintain the mature commercial blue chip as blue light and introduce an ultraviolet chip to excite green and red fluorescent 
materials in order to achieve green and red light. Among these strategies, the core point is that using a short-wavelength light to excite fluorescent materials attains the primary color.

Fluorescent materials are key to improving display performance such as the color gamut. In the past decade, fluorescent materials have evolved from a single YAG:Ce ${ }^{3+}$ phosphor to green and red fluorescent materials such as $\beta$-sialon: $\mathrm{Eu}$ and $\mathrm{CaAlSiN}_{3}: \mathrm{Eu}$ phosphors [9-13]. However, these fluorescent materials with a half-width wider than $50 \mathrm{~nm}$ are not ideal. Further improving the color gamut of LEDs is a hot spot for future commercial competition [14,15]. As a new type of luminescent material, quantum dots (QDs) are quite promising owing to their narrow half-peak width down to $30 \mathrm{~nm}$, wide excitation wavelength, high color purity, and tunable emission [16,17].

LED combined with QDs is an important candidate for next-generation high-quality semiconductor devices [18,19]. By taking the advantages of the QD, a green or red LED could restore the natural color to the greatest extent [20]. Shin-Tson Wu et al. applied CdSe QDs for backlight applications, and a 115\% color gamut in CIE 1931 was attained [21]. Similar high-quality colors from QD were reported by Shinae Jun [22], Jian Chen [23], and Huang-Yu Lin [24] et al. In order to obtain green and red LEDs, QDs are typically excited by short wavelengths such as blue or ultraviolet (UV) light [24,25]. However, the effect of the excitation wavelength on the optical performance of QD devices has not been systematically studied.

Herein, using CdSe QDs, this study compared the performance of green and red QD LEDs at different excitation wavelengths. The photoluminescence (PL) pattern of QD films was explored. Then, the optical performances of green and red LEDs were compared and analyzed at different excitation wavelengths. It turns out the excitation wavelength of the LED chip has an important impact on the overall device performance.

\section{Methods}

\subsection{Materials and Equipments}

The green and red QDs were purchased from Beijing Beida Jubang Science \& Technology Co., Ltd. The PDMS package material was purchased from Dow Corning Corporation, USA. TEM images were taken by a transmission electron microscope (TEM, JEM-2100F, JEOL, Akishima, Japan) operated at an accelerating voltage of $200 \mathrm{kV}$ while dropping the QD solution on the copper network. The PL spectrum was measured by a fluorescence spectrophotometer (RF-6000, Shimadzu, Kyoto, Japan) under $365 \mathrm{~nm}$ of excitation. The absorption and transmission spectra were collected by a UV-vis spectrometer with an integrating sphere accessory (TU-1901, Persee, Beijing, China). The electroluminescence (EL) spectra and optical parameters of the LED devices were measured with a calibrated integrating sphere system, a spectrometer (USB2000+, Ocean Optics, Largo, FL, USA), and a power supply (Keithley 2425, Keithley Instruments \& Products, Cleveland, OH, USA).

\subsection{Characterization of Quantum Dot}

To determine the characteristics of the QDs used in this study, their PL and absorbance spectrum after dispersal in $n$-hexane were measured and are shown in Figure 1. The emission peaks of green QDs (GQDs) and red QDs (RQDs) are located at 525 and $626 \mathrm{~nm}$, while their absorption peaks are at 515 and $610 \mathrm{~nm}$, respectively. This difference between the emission and absorption peaks is attributed to the Stokes shift effect. The half width of the emission peak is $30 \mathrm{~nm}$ for GQDs and $28 \mathrm{~nm}$ for RQDs. This indicates that the QDs have a narrower half-width than most common fluorescent materials such as YAG:Ce ${ }^{3+}$ phosphor $[26,27]$ and that the color purity can be guaranteed. The inset picture also indicates their excellent color purity. QDs can absorb light of a shorter wavelength than their PL emission peaks, and QDs are more capable of absorbing ultraviolet light than in absorbing blue light such as that at $455 \mathrm{~nm}$. 

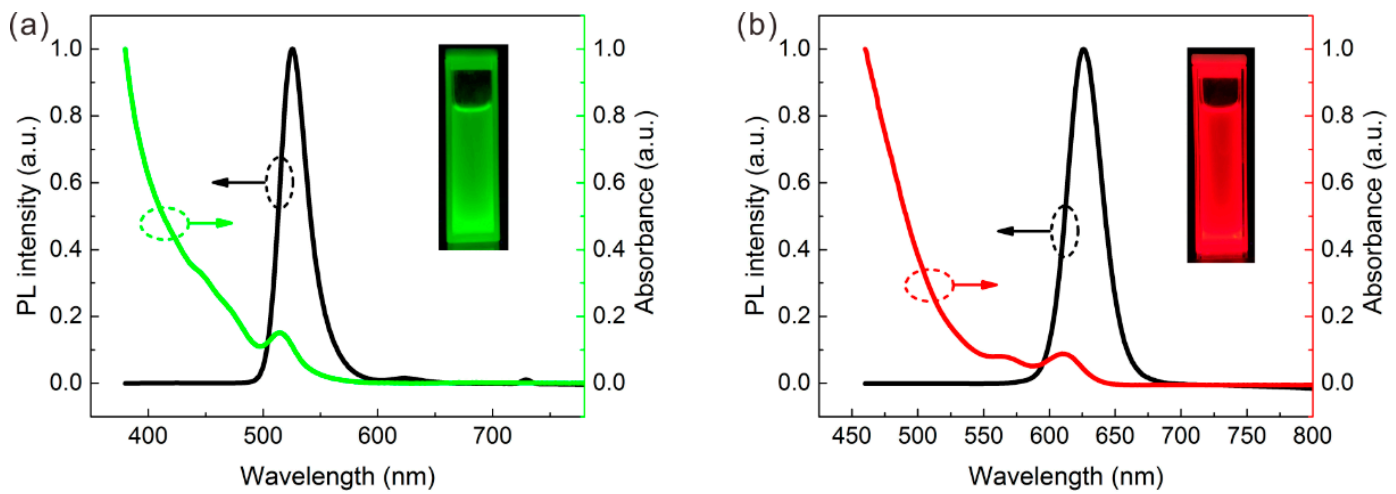

Figure 1. Photoluminescence (PL) and absorbance spectrum of (a) GQDs and (b) RQDs.

From the transmission electron microscope (TEM) graph, all of the QDs show good dispersibility in $n$-hexane. The QDs exhibit a distinct spherical shape, which is formed by the core-shell structure. For these samples, the CdSe acts as a core, and the ZnS acts as a shell. Further, by using ImageJ software to calculate the diameter of 100 QDs in the TEM graph, the particle size distribution is shown in Figure 2. Both particle size distributions show an approximately normal distribution. The average diameter of the GQDs and RQDs is $7.63 \pm 1.16 \mathrm{~nm}$ and $9.55 \pm 1.10 \mathrm{~nm}$, respectively. The particle size of RQDs is larger than that of the GQDs, which is consistent with the reported discovery: the emission peak of CdSe-based QD is related to its particle size, and a larger diameter can result in a longer emission wavelength $[28,29]$.
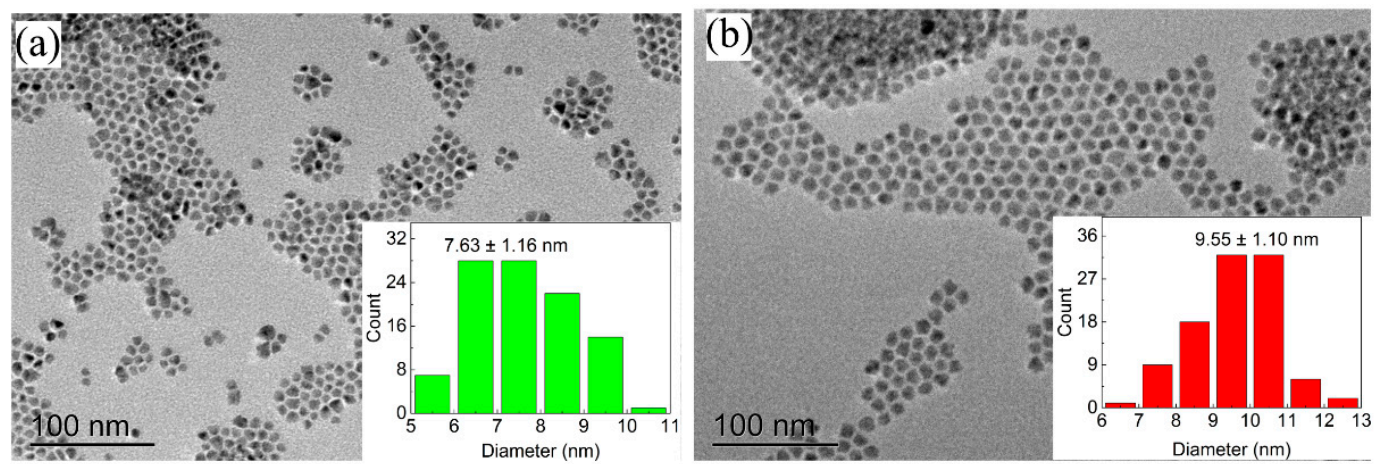

Figure 2. TEM graph of (a) GQDs and (b) RQDs; inset picture shows particle size distribution of QDs.

\subsection{Fabrication of QD-Based LED with Different Excitation Wavelengths}

To obtain a QD-based LED with different excitation wavelengths for this study, first, commercial LED chips of the same size $(45 \times 45 \mathrm{mil})$ with $365,385,405$, and $455 \mathrm{~nm}$ of emission wavelengths were mounted on a copper substrate. The copper substrate serves as a circuit board and a heat sink. Then, the golden wire was connected by ultrasonic welding. For QD applications, the remote excitation to attain green and red light is the basic LED package structure in this study, as shown in Figure 3. Polydimethylsiloxane (PDMS) was selected as the package material. PDMS gel was injected into the cavity and then cured at $100{ }^{\circ} \mathrm{C}$ for $1 \mathrm{~h}$.

To prepare QD remote films for LEDs, green CdSe/ZnS and red CdSe/ZnS/ZnS QDs were selected. After dispersal in $n$-hexane, the QDs were mixed with the PDMS at mass concentrations of $0,0.2,0.5$, $1.2,3.0$, and $7.0 \mathrm{wt} \%$. Then, the film was prepared using a designed mold. The thickness of the film was fixed at $500 \mu \mathrm{m}$ by a gasket. After $1 \mathrm{~h}$ of heating at $100^{\circ} \mathrm{C}$, the mold could be released to obtain QD films. Finally, the QD remote film was assembled to form LED samples with different excitation wavelengths. Green and red QD-converted LEDs (GQD LED and RQD LED) were obtained. 
(a)

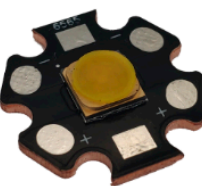

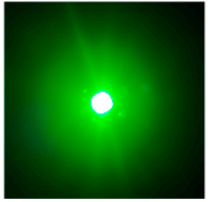

(b)
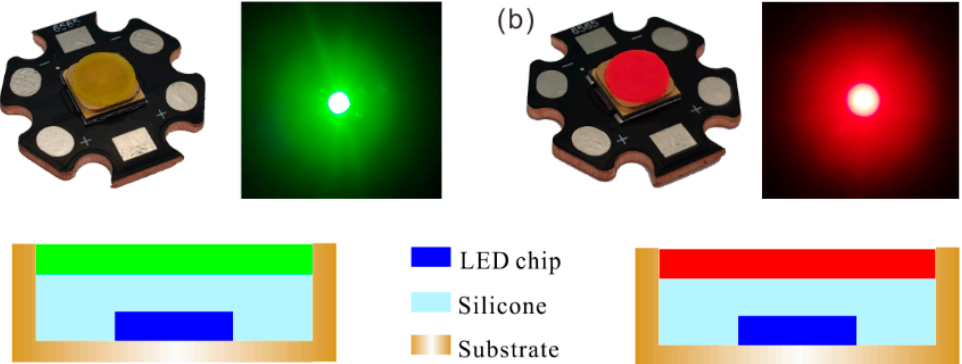

Green QD film

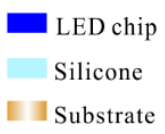

I. Substrate

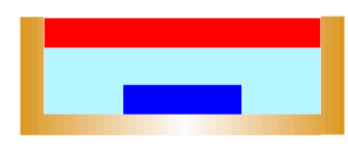

Red QD film

Figure 3. Physical picture and schematic diagram of (a) green quantum-dot LED and (b) red quantum-dot LED.

\section{Results and Discussion}

\subsection{Characterization of $Q D$ Film}

After the QDs were prepared as QD film, the properties of the QD films were explored. As shown in Figure 4a,b, the absorbance peaks of the GQD and RQD films were located at $512 \mathrm{~nm}$ and $610 \mathrm{~nm}$, respectively. These are almost identical to the absorption peak of the QD solution, which means that the film maintains the original luminescence properties of the QDs. The absorbance of both GQD- and RQD-based film increases as the concentration of QDs increases. After adding the QDs, the absorbance below the absorption wavelength increases significantly, and the absorbance of the ultraviolet region is more pronounced than in the blue region. This regular pattern is also reflected in the transmission spectrum, as shown in Figure S1. Thus, with more QDs, more short-wavelength light could be absorbed.
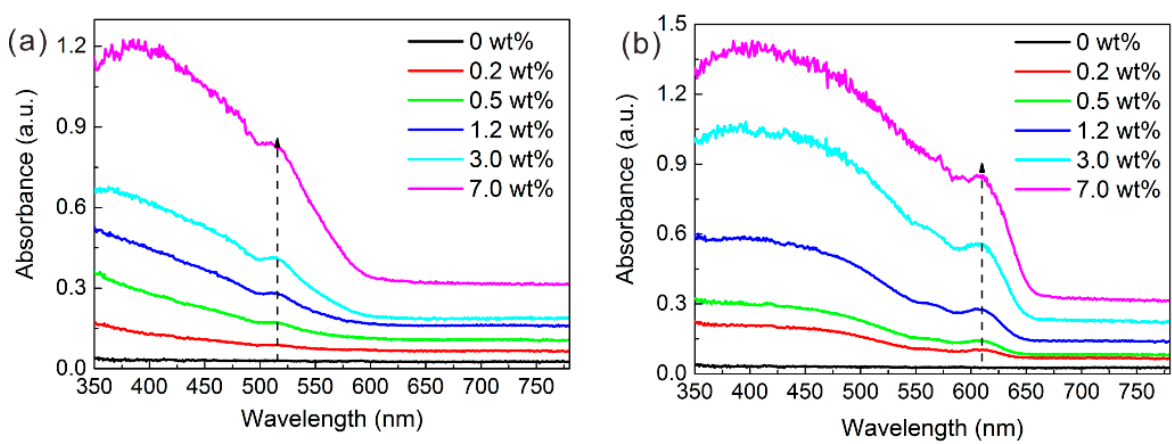

(c)

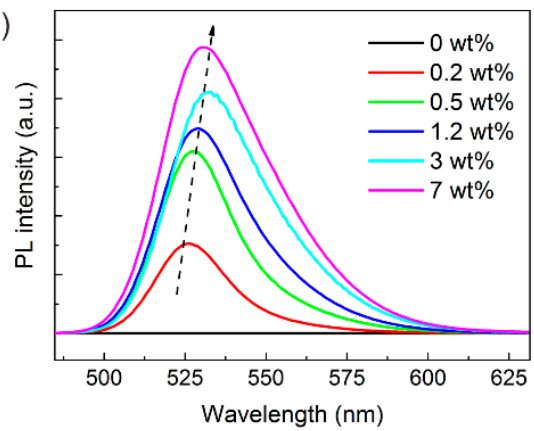

(d)

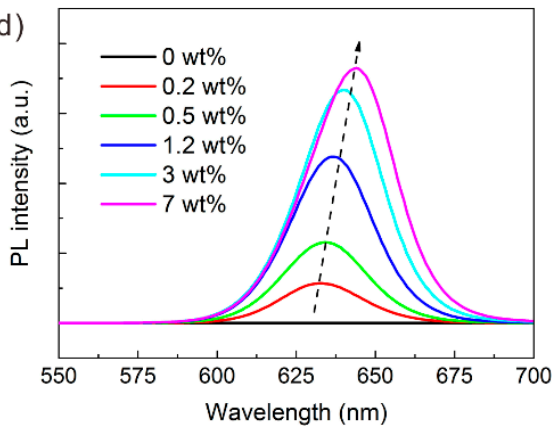

Figure 4. Absorption spectrum of (a) GQD and (b) RQD film; photoluminescence (PL) spectrum of (c) GQDs and (d) RQD film.

The PL spectra of different QD films excited at $365 \mathrm{~nm}$ are shown in Figure 4c,d. The higher the QD concentration, the greater the PL intensity that can be attained. However, a red-shift in the emission peak occurs at a higher QD concentration. For example, at a low concentration of $0.2 \mathrm{wt} \%$, the emission wavelength of GQD film is $526 \mathrm{~nm}$, but at a high concentration of $7 \mathrm{wt} \%$, it is red-shifted to $531 \mathrm{~nm}$, 
as shown in Figure S2. The reason for the red shift could be the agglomeration and reabsorption of QDs. Agglomeration leads to an increase in the average particle size of the QDs, and the QDs have special properties of reabsorption and reemission [30]. Since the red QD absorption is much stronger than the green QD absorption at $365 \mathrm{~nm}$ of excitation, the reabsorption effect is much more significant. Thus, the RQD suffers from a more severe red-shifted effect at high concentrations such as $7.0 \mathrm{wt} \%$.

\subsection{Light Conversion Optical Model of QD LED}

With the combination of the LED chip and QD film, the entire device forms a green or red LED, which is the fundamental base of a display pixel. As shown in Figure 5, LED light transformation can be summarized into three stages:

(1) Absorption. The short-wavelength light from the LED chip is absorbed by the QDs. Thus, the QD absorption rate is defined as the ratio of the absorbed short-wavelength light intensity to the initial short-wavelength light intensity. The QD absorption rate can be used to measure the initial peak elimination ability of QDs for short-wavelength light;

(2) Conversion. Then, the QDs can turn the short-wavelength light into their own emission light. For example, the green QD transforms the blue light into green emissions. Thus, the QD conversion efficiency is defined as the ratio of the QD emission intensity to the absorbed short-wavelength light intensity. The QD conversion efficiency reflects the ability to convert short-wavelength light;

(3) Output. After mixing the residual short-wavelength light intensity and the QD emission peak intensity, the LED devices produce new light output. In other words, the final optical output is composed of residual short-wavelength light and QD emissions. Thus, the energy conversion efficiency is defined as the ratio of the final total light intensity to the initial light intensity. This can reveal the energy utilization of the total LED device.

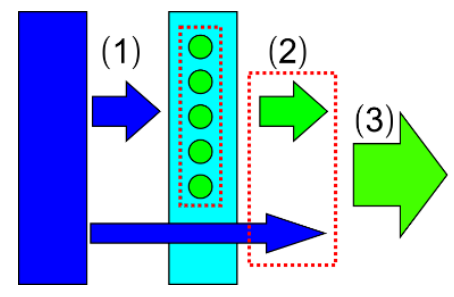

(a)

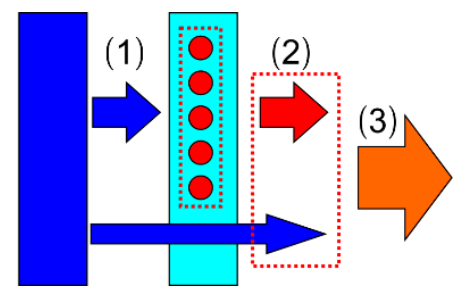

(b)

Figure 5. Light conversion optical models of (a) GQD LED and (b) RQD LED.

\subsection{Performance of GQD LED with Different Excitation Wavelengths}

The EL spectra of different GQD LEDs are explored and shown in Figure 6. At different excitation wavelengths, the following discoveries about QD concentrations are consistent: First, as the concentration of GQD increases, the peak of short-wavelength light decreases while the green peak first rises and then falls. Almost all of the LED samples at different excitation wavelengths show the top green peak intensity at $1.2 \mathrm{wt} \%$ of GQD concentration. Second, the green peak emission wavelength shows a red shift with increasing GQD concentration, which is consistent with the PL test of the QD film. Third, the overall radiant flux of the LED device decreased. This confirms the significant energy loss during QD conversion and this situation becomes more serious with high concentration QDs. Therefore, varieties in QD concentration at different excitation wavelengths show similar patterns in the LED spectrum. This means that even if the initial excitation wavelength of the device is changed, the basic shape of the EL spectrum stays the same.

To further analyze the effects of the excitation wavelength, the optical model described above is introduced here. The first stage is absorption. For the GQD absorption rate, all LEDs with different excitation wavelengths indicate that the higher the QD concentration, the more the short-wavelength light is absorbed, as shown in Figure 7a. However, the GQD absorption rate excited by UV light is 
much higher than that by blue light with the same QD concentration. Only a GQD concentration of $0.2 \mathrm{wt} \%$ can absorb nearly half of the $365 \mathrm{~nm}$ original light intensity (47.7\%). Further, the initial incident radiant flux of LED chips and the QD concentration (1.2 wt\%) were fixed to ensure a single variable of the excitation wavelength. Three initial incident radiant fluxes of the LED chips were studied: 20, 60, and $100 \mathrm{~mW}$. Under a $20 \mathrm{~mW}$ incident radiant flux, the GQDs could absorb 69.5\% of the $365 \mathrm{~nm}$ violet light, while only $53.3 \%$ of the $455 \mathrm{~nm}$ blue light could be absorbed, as shown in Figure $7 \mathrm{~d}$. The ability to absorb short wavelengths of GQD increased by $16.1 \%$ under $365 \mathrm{~nm}$ of excitation. Thus, a solution that combines the UV light and QDs has advantages in eliminating raw chip peaks.
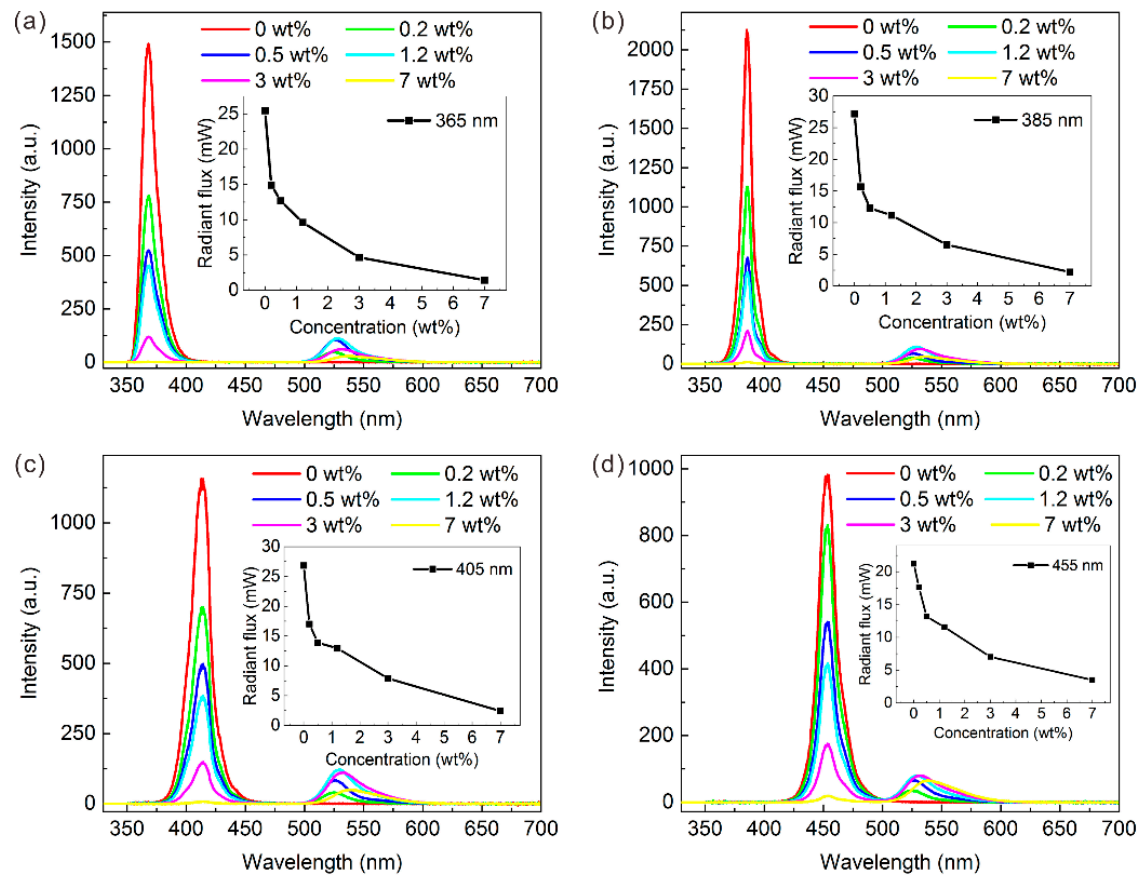

Figure 6. Electroluminescence (EL) spectrum of GQD LED with (a) 365 nm, (b) 385 nm, (c) 405 nm, and (d) $455 \mathrm{~nm}$ of LED-chip excitation wavelength under $20 \mathrm{~mA}$. Inset graph shows radiation flux vs. GQD concentration.
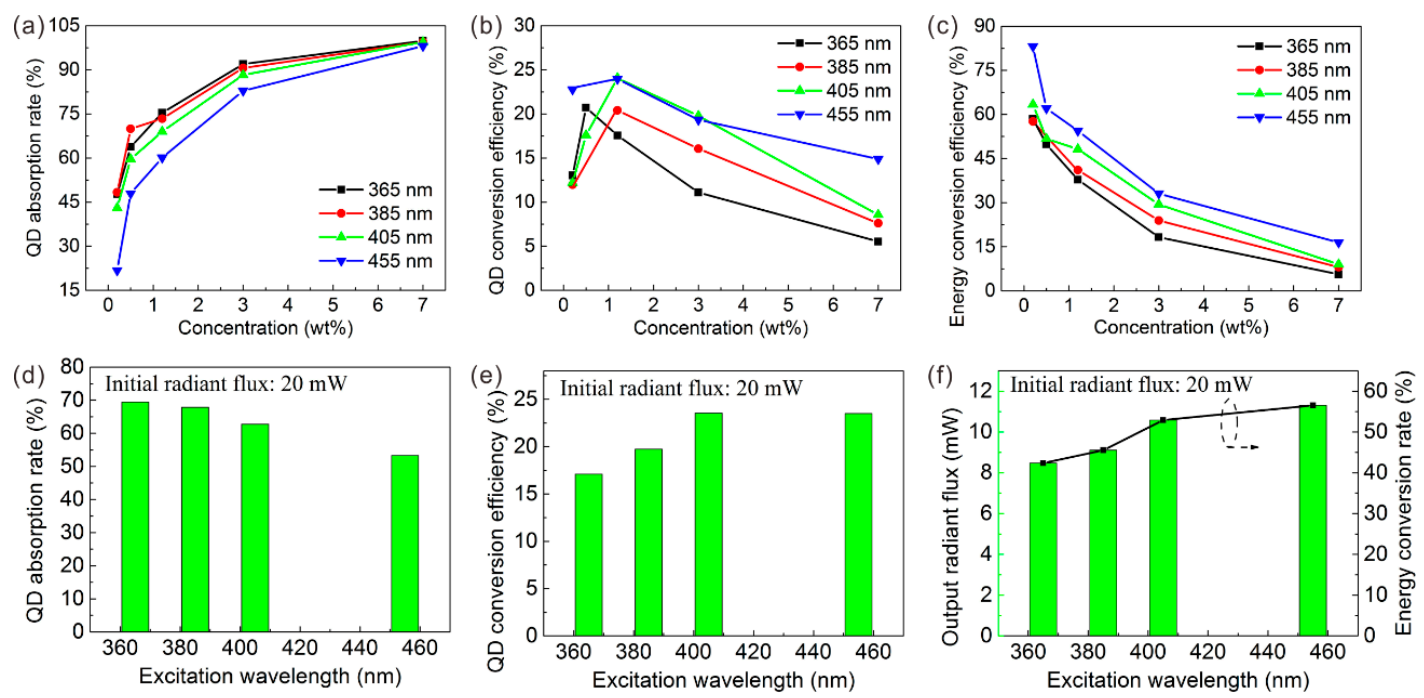

Figure 7. (a,d) QD absorption rate, (b,e) QD conversion efficiency, and (c,f) energy conversion efficiency of GQD LED with different excitation wavelengths. 
Then, the absorbed short-wavelength light is converted to green light by GQDs. Among the excitation wavelengths, the QD conversion efficiency of GQD rises first and then drops as the QD concentration increases. Thus, a moderate QD concentration such as $1.2 \mathrm{wt} \%$ can maximize the QD conversion efficiency. The QD conversion efficiency tends to increase at longer excitation wavelengths. In other words, the QD conversion efficiency excited by ultraviolet light is lower than that by blue light. Taking the $20 \mathrm{~mW}$ incident radiant flux as an example, the QD conversion efficiency is only $17.1 \%$ under $365 \mathrm{~nm}$ of excitation but increases to $23.5 \%$ with $455 \mathrm{~nm}$ of excitation, a $6.4 \%$ increase. It should be pointed out that the QD conversion efficiency is not ideal; this could be owing to the severe reabsorption characteristics of QDs [31]. In short, typical blue light has an advantage in QD conversion efficiency.

The last part of the optical model is the output. At different excitation wavelengths, the energy conversion efficiency of all LEDs declines as the QD concentration increases. This may be owing to the agglomeration and reabsorption effect of the high QD concentration, which is more likely to produce significant heat [32,33]. Therefore, from the perspective of energy utilization, a low QD concentration is better for LED applications. However, at the same QD concentration, the energy conversion efficiency gradually increases as the excitation wavelength increases. The typical blue $455 \mathrm{~nm}$ excited LED has a maximum energy conversion efficiency of $83.1 \%$ with a GQD concentration of $0.2 \mathrm{wt} \%$. As shown in Figure 7f, when the incident radiant flux is $20 \mathrm{~mW}$, the output radiant flux of the LED with excitation wavelengths of $365,385,405$, and $455 \mathrm{~nm}$ is $8.5,9.1,10.6$, and $11.3 \mathrm{~mW}$, respectively. Therefore, the energy conversion efficiency at $455 \mathrm{~nm}$ is $14.1 \%$ higher than that at $365 \mathrm{~nm}$. Repeated experiments with different incident radiant fluxes such as 60 and $100 \mathrm{~mW}$ demonstrate the reliability of the above conclusions, as shown in Figure S3.

To determine the color quality of these different LEDs, their color coordinates are summarized and drawn in the chromaticity diagram in Figure 8. The colorimetric behavior of the GQD LED with different excitations can be divided into two categories: (A) 365 and $385 \mathrm{~nm}$ of excitation and (B) 405 and $455 \mathrm{~nm}$ of excitation. Under 365 and $385 \mathrm{~nm}$ of excitation, the $x$ color coordinate keeps increasing, while the $y$ color coordinate first increases and then decreases as the GQD concentration increases. This indicates that the green light gradually plays a leading role. The color coordinates only shifted within the green-light area. Interestingly, the LED excited at $365 \mathrm{~nm}$ at a low QD concentration of $0.2 \mathrm{wt} \%$ emits a relatively high-purity green light. However, 405 and $455 \mathrm{~nm}$-based LEDs show distinct color qualities. Both the $x$ and $y$ color coordinates keep increasing as the QD concentration increases, which leads to a gradual light shift from the blue area to the green area. Even the green color quality of the $455 \mathrm{~nm}$ LED with the highest QD concentration at $7.0 \mathrm{wt} \%$ could not compete with the $365 \mathrm{~nm}$ LED with the lowest QD concentration at $0.2 \mathrm{wt} \%$. If the LEDs with different excitation wavelengths aim to obtain the same green light color quality, the GQD concentration at $455 \mathrm{~nm}$ of excitation should be much higher than that at $365 \mathrm{~nm}$ of excitation. Thanks to the excellent color characteristics of the QD itself, the color coordinate of the GQD LED with $365 \mathrm{~nm}$ excitation and high GQD concentration at $7.0 \mathrm{wt} \%$ is $(0.322,0.663)$. This green light almost reaches the edge of the chromaticity diagram, which means that ultrawide color gamut performance is possible if this GQD is applied to displays.

Even with the same incident light power and the same GQD concentration, the LEDs clearly exhibit different color properties owing to different excitation wavelengths, as shown in Figure 9. The color coordinates of the green LED excited at $365,385,405$, and $455 \mathrm{~nm}$ are $(0.231,0.706),(0.230$, $0.674),(0.202,0.318)$, and $(0.162,0.123)$ under a $20 \mathrm{~mW}$ incident radiant flux, respectively. Thus, both the $x$ and $y$ coordinates show a downward trend. In other words, under the excitation of a $365 \mathrm{~nm}$ LED chip, the entire GQD device emits high-purity green light. However, under the excitation of the 455-nm LED chip, the device emits blue light. This superiority in color purity excited at $365 \mathrm{~nm}$ is mainly owing to two reasons: the GQD LEDs excited at $365 \mathrm{~nm}$ have unique advantages in eliminating initial short peaks owing to the stronger absorption ability, and the naked eye is not sensitive to residual $365 \mathrm{~nm}$ ultraviolet light. But green light from QD emission is visible and the human eye is quite sensitive to green light. Thus, in terms of color purity, the excitation of QDs by ultraviolet light such as 
$365 \mathrm{~nm}$ can maximize the advantages of the quantum-dot color quality. This UV excitation solution is recommended for display applications.

(a)

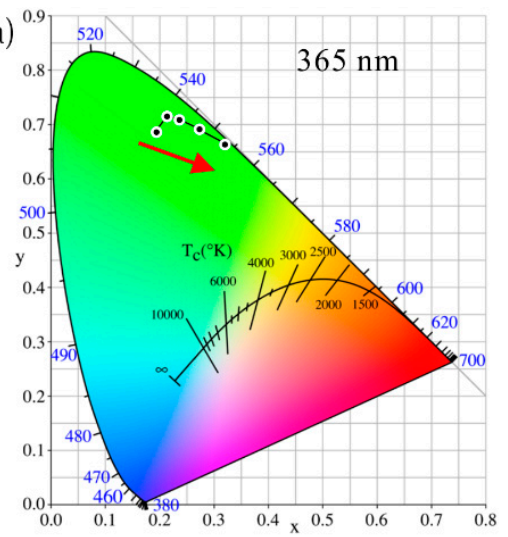

(c)

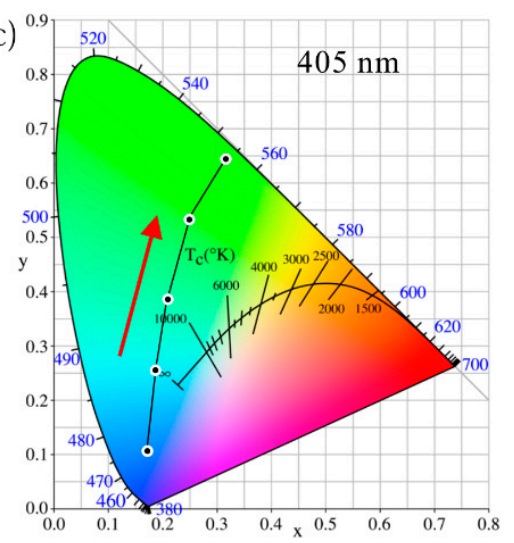

(b)

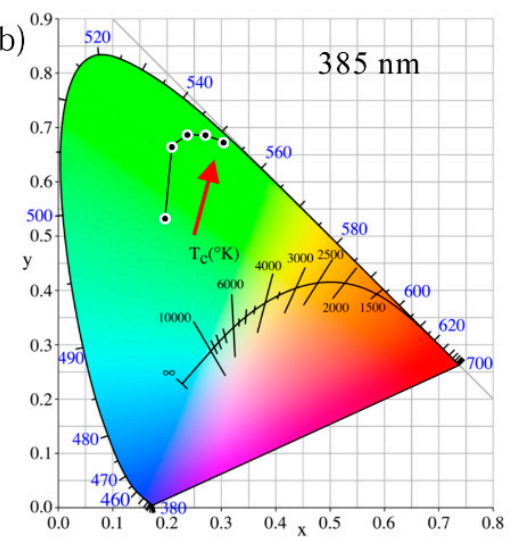

(d)

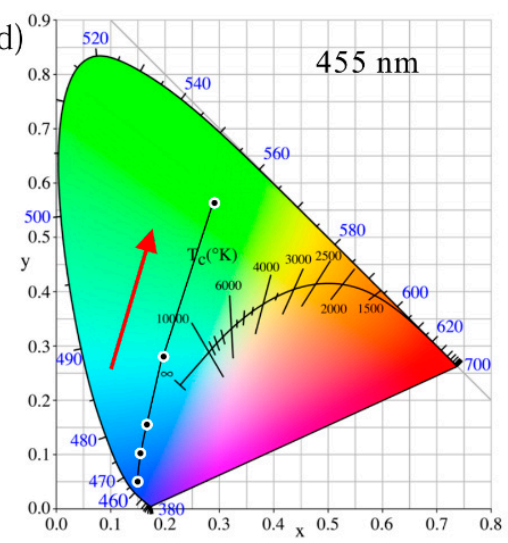

Figure 8. Chromaticity diagram of GQD LED with different QD concentrations under (a) $365 \mathrm{~nm}$, (b) $385 \mathrm{~nm}$, (c) $405 \mathrm{~nm}$, and (d) $455 \mathrm{~nm}$ of excitation.
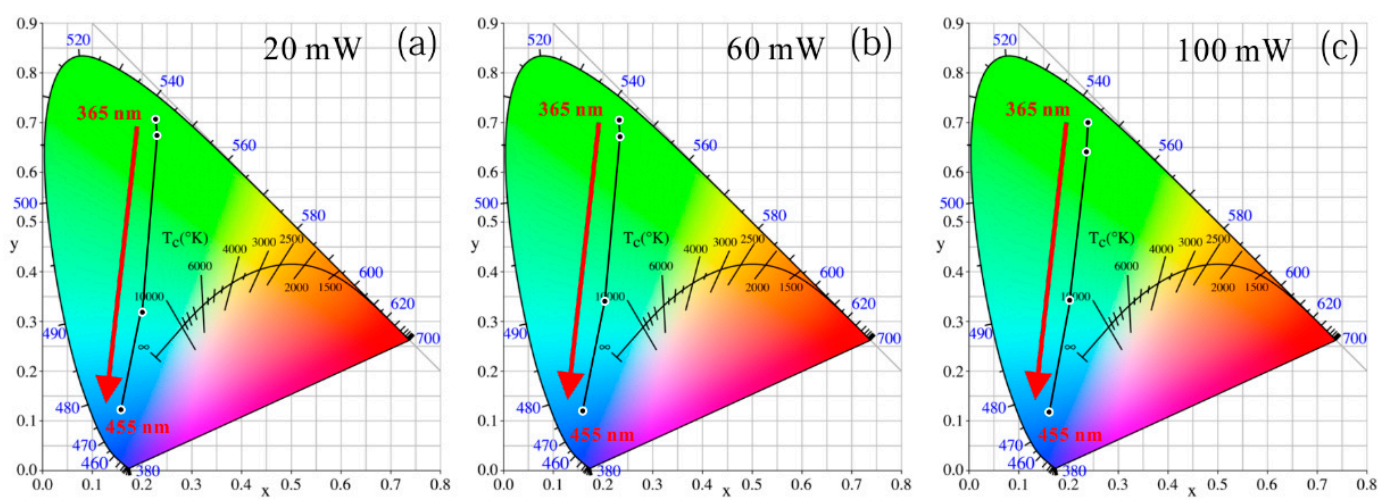

Figure 9. Chromaticity diagram of GQD LED with different excitation wavelengths under equal incident radiant fluxes of (a) $20 \mathrm{~mW}$, (b) $60 \mathrm{~mW}$, and (c) $100 \mathrm{~mW}$.

\subsection{Performance of RQD LEDs with Different Excitation Wavelengths}

As for the RQDs, the EL spectrum is also explored and shown in Figure S4. The EL spectra reveal that LEDs based on RQDs exhibit laws similar to those of GQDs. Then, as with the GQD LED, the QD absorption rate, QD conversion efficiency, and energy conversion efficiency of the RQD LED were calculated and are shown in Figure 10. In general, the red LED has a pattern similar to that of the green LED. Unlike green QDs, almost all of the short-wavelength light can be absorbed with only $3.0 \mathrm{wt} \%$ concentration RQDs. This reveals that RQDs have a stronger advantage than GQDs in eliminating short peaks. 
Under $20 \mathrm{~mW}$ of incident optical power, the RQD absorption rate excited at $365 \mathrm{~nm}$ is $5.0 \%$ higher than that at $455 \mathrm{~nm}$, but the RQD conversion efficiency and energy conversion efficiency excited at $455 \mathrm{~nm}$ are $14.3 \%$ and $15.5 \%$ higher, respectively, than that at $365 \mathrm{~nm}$. Like the GQD, the blue chip is recommended from the perspective of energy utilization because the maximum energy conversion efficiency is $69.3 \mathrm{wt} \%$ from the $455 \mathrm{~nm}$ LED. At different incident power such as 60 and $100 \mathrm{~mW}$, the same law is exhibited in Figure S5.
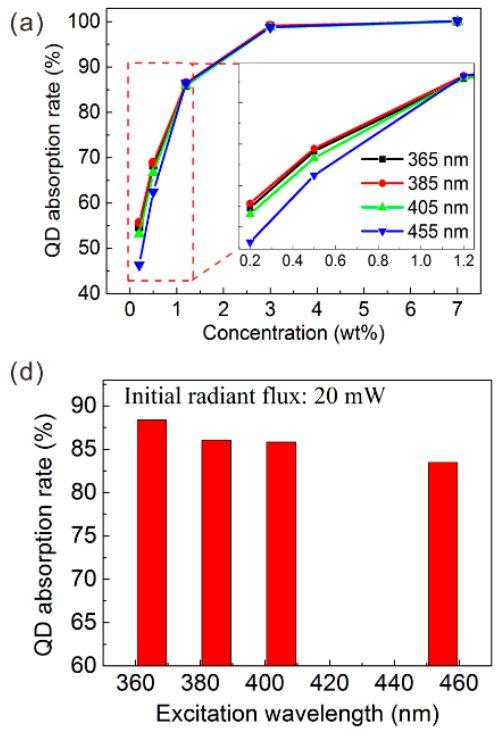
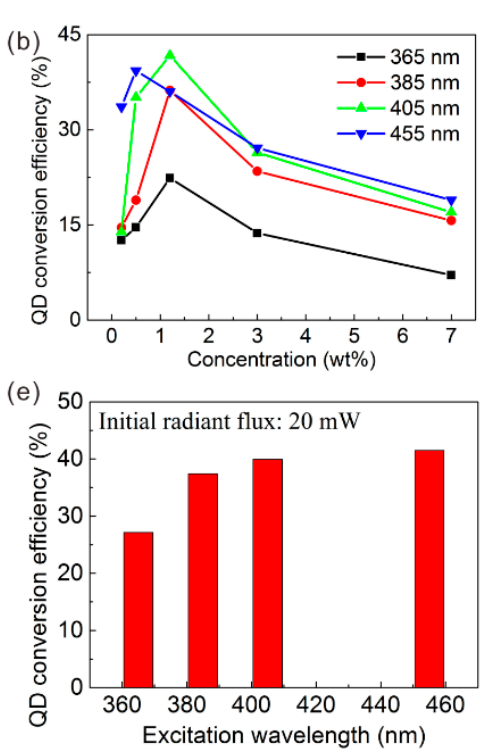
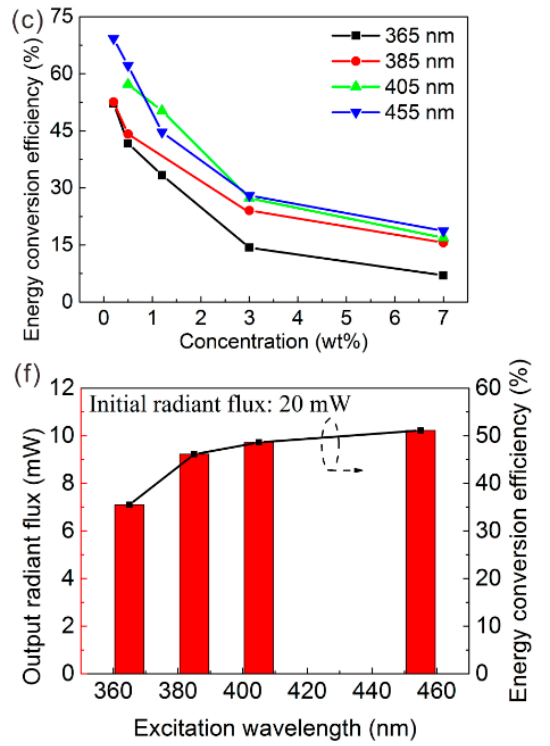

Figure 10. (a,d) QD absorption rate, (b,e) QD conversion efficiency, and (c,f) energy conversion efficiency of GQD LED with different excitation wavelengths.

A chromaticity diagram of the RQD LED under different wavelength excitations is also summarized. There is a significant difference in behavior in the color performance between a typical UV $365 \mathrm{~nm}$ LED and a blue $455 \mathrm{~nm}$ LED. Under $365 \mathrm{~nm}$ of excitation, because the original peak from the LED chip is more easily eliminated owing to the stronger absorption ability and the human eye is not sensitive to residual $365 \mathrm{~nm}$ ultraviolet light. The main color perceived by the human eye is visible red light, which leads to excellent red-light quality even at a low QD concentration of $0.2 \mathrm{wt} \%$. In addition, the red color quality of the $385 \mathrm{~nm}$ excitation is similar to that of $365 \mathrm{~nm}$ LED but is slightly closer to the orange color at low concentrations. However, under $455 \mathrm{~nm}$ of excitation, the color shifts from blue and purple to red. The LED exhibits significant blue light at low concentrations such as $0.2 \mathrm{wt} \%$. The $405 \mathrm{~nm}$ LED shows patterns similar to those of the blue excitation.

Even with the same incident light power and the same RQD concentration (1.2 $\mathrm{wt} \%)$, the color performance of these LED samples is totally different, as shown in Figure 11. From $365 \mathrm{~nm}$ to $455 \mathrm{~nm}$ of excitation, the color shifts from red to purple regions, as shown in Figure S6. Taking advantage of the QD, the color coordinates of the $365 \mathrm{~nm}$ excitation basically reached the boundaries of the gamut, which means that the red color is highly pure. These experimental results reveal that the combination of UV light with RQDs has unparalleled advantages in colorimetry, which may shine in future display fields. Therefore, the red LED prepared by ultraviolet light to excite RQDs is superior to the blue-light excitation in attaining high-quality red light. This is consistent with the conclusion regarding GQDs. 
(a)

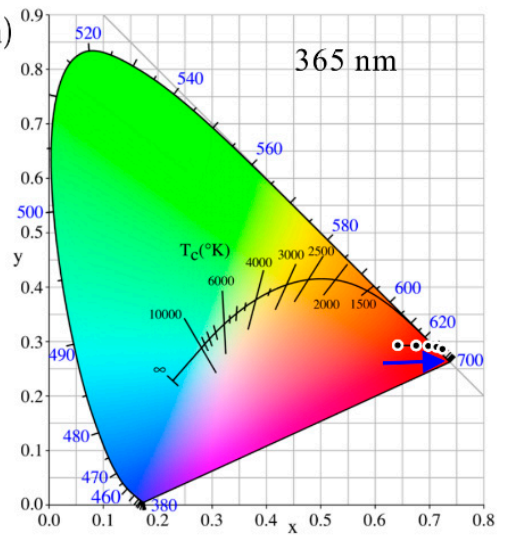

(c)

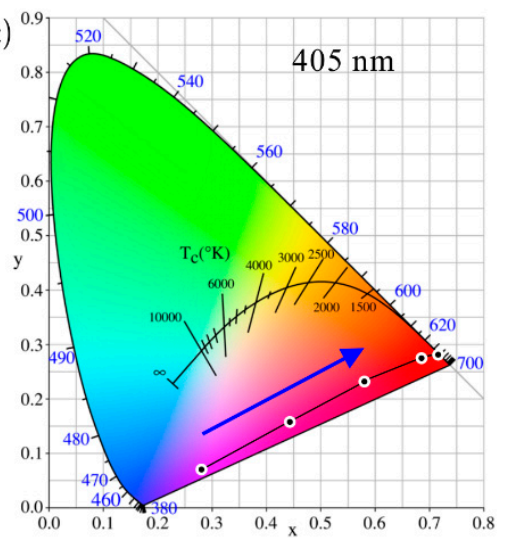

(b)

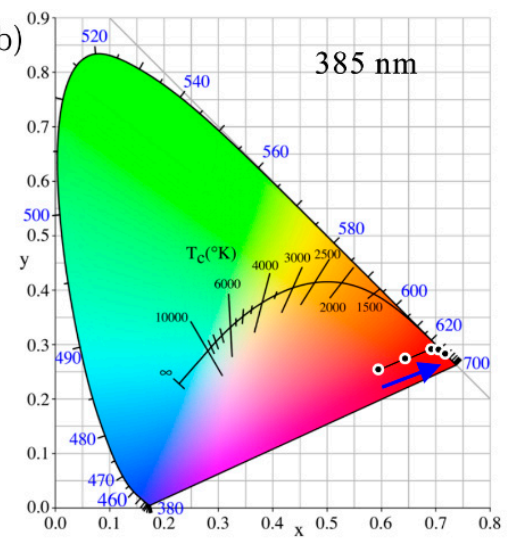

(d)

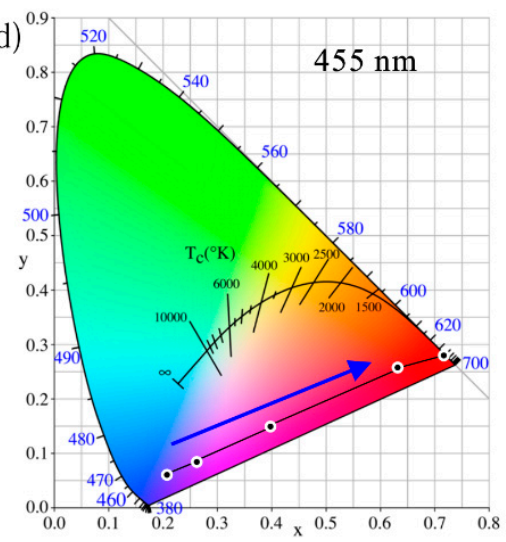

Figure 11. Chromaticity diagram of RQD LED with different QD concentrations under (a) $365 \mathrm{~nm}$, (b) $385 \mathrm{~nm}$, (c) $405 \mathrm{~nm}$, and (d) $455 \mathrm{~nm}$ of excitation.

\subsection{Comparison of Color Gamut between Blue (455 nm) Excitation and Ultraviolet (365 nm) Excitation Solutions}

The color gamut is an important parameter when evaluating RGB display systems. The wider the gamut, the higher the fidelity of the colors of nature that can be attained. A comparison of the color gamut for different excitation wavelength solutions is analyzed in Table 1. The blue-color coordinates of all solutions are derived from the blue LED chip, and the green and red color coordinates are obtained from the QD-converted LEDs. With the highest QD concentration at 7.0\%, the RGB color gamut excited at $455 \mathrm{~nm}$ is only $84.9 \%$ of the NTSC1953 standard. Interestingly, the color gamut of 365 nm-excited solution could cover $99.7 \%$ of the NTSC1953 standard area even at a low QD concentration $(0.2 \mathrm{wt} \%)$. Moreover, as shown in Figure 12, the color gamut of a 365-nm-excited RGB system could be high as $117.5 \%$ by adjusting the QD concentration properly. This is a $32.6 \%$ improvement over the blue-light-excited solution. Thus, the UV-excited quantum-dot solution can achieve a wider color gamut and has great potential for development in quantum-dot applications, especially in the display field. 


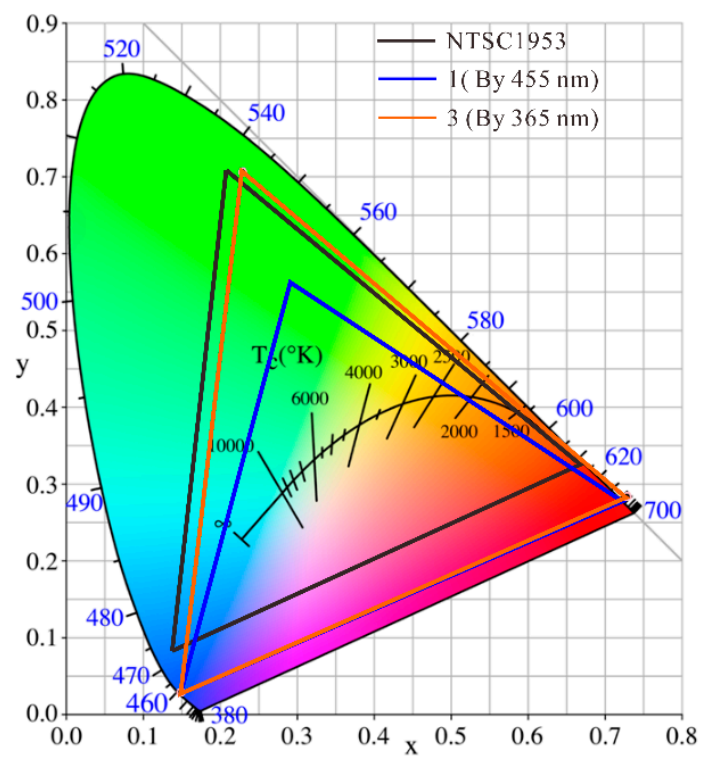

Figure 12. Comparison of color gamut between blue (455-nm) excitation and ultraviolet (365-nm) excitation solutions.

Table 1. Comparison of color gamut for wavelength solutions with different excitations.

\begin{tabular}{cccccccc}
\hline Group & $\begin{array}{c}\text { Excitation } \\
\text { Wavelength }(\mathbf{n m})\end{array}$ & $\begin{array}{c}\text { GQD } \\
\mathbf{( \% )}\end{array}$ & $\begin{array}{c}\text { RQD } \\
\mathbf{( \% )}\end{array}$ & $\mathbf{R}(\boldsymbol{x}, \boldsymbol{y})$ & $\mathbf{G}(\boldsymbol{x}, \boldsymbol{y})$ & $\begin{array}{c}\text { NTSC1953 } \\
\text { Standard }(\boldsymbol{\%})\end{array}$ \\
\hline 1 & 455 & 7.0 & 7.0 & $(0.7210,0.2794)$ & $(0.2935,0.5624)$ & $(0.1492,0.0298)$ & 84.9 \\
2 & 365 & 0.2 & 0.2 & $(0.6485,0.2931)$ & $(0.1947,0.6855)$ & $(0.1492,0.0298)$ & 99.7 \\
3 & 365 & 1.2 & 7.0 & $(0.7289,0.2834)$ & $(0.2300,0.7064)$ & $(0.1492,0.0299)$ & 117.5 \\
\hline
\end{tabular}

\section{Conclusions}

In this study, we investigated the effect of the excitation wavelength on the optical performances of QD-converted light-emitting diodes. Sample LEDs with excitation light sources of 365, 385, 405, and $455 \mathrm{~nm}$ were prepared, and their optical performances were compared. Under different excitation wavelengths, green and red QD-converted LEDs showed similar patterns. The QD conversion efficiency and energy conversion efficiency increase at longer excitation wavelengths. At an incident radiant flux of $20 \mathrm{~mW}$, the QD conversion efficiency and energy conversion efficiency of the $455 \mathrm{~nm}$-excited GQD LED were $6.4 \%$ and $14.1 \%$ higher than those of the $365 \mathrm{~nm}$ LED. Therefore, blue light is recommended to excite QDs with regard to energy utilization. However, the UV-excited QD LED solution has more advantages than blue-light excitation for color purity. Even for the $455 \mathrm{~nm}$ LED with the highest QD concentration at $7.0 \mathrm{wt} \%$, the green color quality could not compete with a $365 \mathrm{~nm}$ LED with the lowest QD concentration of $0.2 \mathrm{wt} \%$. This is because QD LEDs excited at $365 \mathrm{~nm}$ have unique advantages in eliminating short peaks because of higher absorption in the ultraviolet range of QD. Moreover, the naked eye is not sensitive to the residual $365 \mathrm{~nm}$ ultraviolet light. As a result, green and red light excited at $365 \mathrm{~nm}$ with a $7.0 \mathrm{wt} \%$ QD concentration can approach the boundary of the chromaticity diagram. Taking advantage of the $365 \mathrm{~nm}$-excited solution, a 117.5\% color gamut of the RGB system could be obtained compared with the NTSC1953 standard, which is an improvement of $32.6 \%$ over the blue-light-excited solution. This research helps understand the variation of quantum-dot-converted LEDs at different excitation wavelengths, and provides basic guidance for the selection of a pumping source for QDs.

Supplementary Materials: The following are available online at http://www.mdpi.com/2079-4991/9/8/1100/s1, Figure S1: Transmission spectrum of (a) GQD and (b) RQD film; Figure S2: PL shift phenomenon of (a) GQD film and (c) RQD film; Figure S3: (a,d) GQD absorption rate; (b,e) GQD conversion efficiency; (c,f) output radiant flux and energy conversion efficiency under 60- and 100-mW incident radiant flux; Figure S4: Electroluminescence (EL) spectrum of RQD LED with (a) $365 \mathrm{~nm}$, (b) $385 \mathrm{~nm}$, (c) $405 \mathrm{~nm}$, and (d) 455-nm LED chip excitation wavelength 
under 20 mA; Figure S5: (a,d) RQD absorption rate; (b,e) RQD conversion efficiency; $(\mathrm{c}, \mathrm{f})$ output radiant flux and energy conversion efficiency under 60- and 100-mW incident radiant flux; Figure S6: Chromaticity diagram of RQD LED with different excitation wavelengths under equal incident radiant fluxes such as (a) $20 \mathrm{~mW}$, (b) $60 \mathrm{~mW}$, and (c) $100 \mathrm{~mW}$.

Author Contributions: Formal analysis, C.Y. and X.D.; funding acquisition, Y.T. and Z.L.; investigation, X.D. and J.L.; methodology, C.Y. and X.D.; resources, J.L. and Z.L.; writing (original draft), C.Y.; writing (review \& editing), Z.L. and Y.T.

Funding: This study was supported by the Science \& Technology Program of Guangdong Province (2019B010130001), the National Natural Science Foundation of China (No. 51775199, No. 51735004) and the Natural Science Foundation of Guangdong Province (2018B030306008).

Conflicts of Interest: The authors declare no conflict of interest.

\section{References}

1. Pimputkar, S.; Speck, J.S.; Denbaars, S.P.; Nakamura, S. Prospects for LED lighting. Nat. Photonics 2009, 3, 180-182. [CrossRef]

2. Nann, T.; Skinner, W.M. Quantum dots for electro-optic devices. ACS Nano 2011, 5, 5291-5295. [CrossRef] [PubMed]

3. Hasan, J.; Ang, S.S. A RGB-driver for LED display panels. In Proceedings of the 2010 Twenty-Fifth Annual IEEE Applied Power Electronics Conference and Exposition (APEC), Palm Springs, CA, USA, 21-25 February 2010; pp. 750-754.

4. Nguyen, F. Challenges in the design of a RGB LED display for indoor applications. Synth. Met. 2001, 122, 215-219. [CrossRef]

5. Li, Z.; Cao, K.; Li, J.; Tang, Y.; Xu, L.; Ding, X.; Yu, B. Investigation of Light-Extraction Mechanisms of Multiscale Patterned Arrays With Rough Morphology for GaN-Based Thin-Film LEDs. IEEE Access 2019, 7, 73890-73898. [CrossRef]

6. Kurtin, J.; Puetz, N.; Theobald, B.; Stott, N.; Osinski, J. 12.5L: Late-News Paper: Quantum Dots for High Color Gamut LCD Displays using an On-Chip LED Solution. SID Symp. Dig. Tech. Pap. 2015, 45, 146-148. [CrossRef]

7. Chen, C.J.; Lien, J.Y.; Wang, S.L.; Chiang, R.K. P-91: Highly-Efficient LEDs with On-Chip Quantum-Dot Package for Wide Color Gamut LCD Display. In Proceedings of the SID Symposium Digest of Technical Papers, San Francisco, CA, USA, 22-27 May 2016; pp. 1465-1468.

8. Peng, Y.; Wang, S.; Li, R.; Li, H.; Cheng, H.; Chen, M.; Liu, S. Luminous efficacy enhancement of ultraviolet-excited white light-emitting diodes through multilayered phosphor-in-glass. Appl. Opt. 2016, 55, 4933-4938. [CrossRef] [PubMed]

9. Anandan, M. Progress of LED backlights for LCDs. J. Soc. Inf. Disp. 2008, 16, 287-310. [CrossRef]

10. Xie, R.J.; Hirosaki, N.; Takeda, T. Wide color gamut backlight for liquid crystal displays using three-band phosphor-converted white light-emitting diodes. Appl. Phys. Express 2009, 2, 022401. [CrossRef]

11. Wang, L.; Wang, X.; Kohsei, T.; Yoshimura, K.I.; Izumi, M.; Hirosaki, N.; Xie, R.J. Highly efficient narrow-band green and red phosphors enabling wider color-gamut LED backlight for more brilliant displays. Opt. Express 2015, 23, 28707-28717. [CrossRef] [PubMed]

12. Nitti, A.; Bianchi, G.; Po, R.; Swager, T.M.; Pasini, D. Domino Direct Arylation and Cross-Aldol for Rapid Construction of Extended Polycyclic $\pi$-Scaffolds. J. Am. Chem. Soc. 2017, 139, 8788-8791. [CrossRef] [PubMed]

13. Pasini, D.; Takeuchi, D. Cyclopolymerizations: Synthetic Tools for the Precision Synthesis of Macromolecular Architectures. Chem. Rev. 2018, 118, 8983-9057. [CrossRef] [PubMed]

14. Chen, H.W.; Zhu, R.D.; He, J.; Duan, W.; Hu, W.; Lu, Y.Q.; Li, M.C.; Lee, S.L.; Dong, Y.J.; Wu, S.T. Going beyond the limit of an LCD's color gamut. Light Sci. Appl. 2017, 6, e17043. [CrossRef] [PubMed]

15. Chen, H.; He, J.; Wu, S.T. Recent Advances on Quantum-Dot-Enhanced Liquid-Crystal Displays. IEEE J. Sel. Top. Quantum Electron. 2017, 23, 1-11. [CrossRef]

16. Song, W.S.; Yang, H. Efficient White-Light-Emitting Diodes Fabricated from Highly Fluorescent Copper Indium Sulfide Core/Shell Quantum Dots. Chem. Mater. 2012, 24, 1961-1967. [CrossRef] 
17. Rao, L.; Tang, Y.; Yan, C.; Li, J.; Zhong, G.; Tang, K.; Yu, B.; Li, Z.; Zhang, J.Z. Tuning the emission spectrum of highly stable cesium lead halide perovskite nanocrystals through poly (lactic acid)-assisted anion-exchange reactions. J. Mater. Chem. C 2018, 6, 5375-5383. [CrossRef]

18. Li, J.; Tang, Y.; Li, Z.; Ding, X.; Yuan, D.; Yu, B. Study on Scattering and Absorption Properties of Quantum-Dot-Converted Elements for Light-Emitting Diodes Using Finite-Difference Time-Domain Method. Materials 2017, 10, 1264. [CrossRef] [PubMed]

19. Tang, Y.; Lu, H.; Li, J.; Li, Z.; Du, X.; Ding, X.; Yu, B. Improvement of Optical and Thermal Properties for Quantum Dots WLEDs by Controlling Layer Location. IEEE Access 2019, 7, 77642-77648. [CrossRef]

20. Coe-Sullivan, S.; Liu, W.; Allen, P.; Steckel, J.S. Quantum dots for LED downconversion in display applications. ECS J. Solid State Sci. Technol. 2013, 2, R3026-R3030. [CrossRef]

21. Luo, Z.; Chen, Y.; Wu, S.T. Wide color gamut LCD with a quantum dot backlight. Opt. Express 2013, 21, 26269-26284. [CrossRef]

22. Jang, E.; Jun, S.; Jang, H.; Lim, J.; Kim, B.; Kim, Y. White-light-emitting diodes with quantum dot color converters for display backlights. Adv. Mater. 2010, 22, 3076-3080. [CrossRef]

23. Chen, J.; Hartlove, J.; Hardev, V.; Yurek, J.; Lee, E.; Gensler, S. P-119: High efficiency LCDs using quantum dot enhancement films. In Proceedings of the SID Symposium Digest of Technical Papers, San Diego, CA, USA, 1-6 June 2014; pp. 1428-1430.

24. Lin, H.Y.; Sher, C.W.; Hsieh, D.H.; Chen, X.Y.; Chen, H.M.P.; Chen, T.M.; Lau, K.M.; Chen, C.H.; Lin, C.C.; Kuo, H.C. Optical cross-talk reduction in a quantum-dot-based full-color micro-light-emitting-diode display by a lithographic-fabricated photoresist mold. Photonics Res. 2017, 5, 411-416. [CrossRef]

25. Han, H.V.; Lin, H.Y.; Lin, C.C.; Chong, W.C.; Li, J.R.; Chen, K.J.; Yu, P.; Chen, T.M.; Chen, H.M.; Lau, K.M. Resonant-enhanced full-color emission of quantum-dot-based micro LED display technology. Opt. Express 2015, 23, 32504-32515. [CrossRef]

26. Loo, K.H.; Lai, Y.M.; Tan, S.C.; Chi, K.T. On the Color Stability of Phosphor-Converted White LEDs Under DC, PWM, and Bilevel Drive. IEEE Trans. Power Electron. 2012, 27, 974-984. [CrossRef]

27. Ding, X.; Li, M.; Li, Z.; Tang, Y.; Xie, Y.; Tang, X.; Fu, T. Thermal and optical Investigations of a laser-driven phosphor converter coated on a heat pipe. Appl. Therm. Eng. 2019, 148, 1099-1106. [CrossRef]

28. Dabbousi, B.O.; Rodriguez-Viejo, J.; Mikulec, F.V.; Heine, J.R.; Mattoussi, H.; Ober, R.; Jensen, K.F.; Bawendi, M.G. (CdSe) ZnS core-shell quantum dots: Synthesis and characterization of a size series of highly luminescent nanocrystallites. J. Phys. Chem. B 1997, 101, 9463-9475. [CrossRef]

29. Zhang, K.; Chang, H.; Fu, A.; Alivisatos, A.P.; Yang, H. Continuous distribution of emission states from single CdSe/ZnS quantum dots. Nano Lett. 2006, 6, 843-847. [CrossRef]

30. Li, J.S.; Tang, Y.; Li, Z.T.; Cao, K.; Yan, C.M.; Ding, X.R. Full spectral optical modeling of quantum-dotconverted elements for light-emitting diodes considering reabsorption and reemission effect. Nanotechnology 2018, 29, 295707. [CrossRef]

31. Li, J.S.; Tang, Y.; Li, Z.T.; Ding, X.R.; Rao, L.S.; Yu, B.H. Effect of quantum dot scattering and absorption on the optical performance of white light-emitting diodes. IEEE Trans. Electron Devices 2018, 65, 2877-2884. [CrossRef]

32. Woo, J.Y.; Kim, K.N.; Jeong, S.; Han, C.S. Thermal behavior of a quantum dot nanocomposite as a color converting material and its application to white LED. Nanotechnology 2010, 21, 495704. [CrossRef]

33. Li, Z.; Song, C.; Qiu, Z.; Li, J.; Cao, K.; Ding, X.; Tang, Y. Study on the Thermal and Optical Performance of Quantum Dot White Light-Emitting Diodes Using Metal-Based Inverted Packaging Structure. IEEE Trans. Electron Devices 2019, 66, 3020-3027. [CrossRef]

(C) 2019 by the authors. Licensee MDPI, Basel, Switzerland. This article is an open access article distributed under the terms and conditions of the Creative Commons Attribution (CC BY) license (http://creativecommons.org/licenses/by/4.0/). 